\title{
MS38-01 | Recent Advances in High-Z Pixel Detectors
}

ruat, marie (ESRF, grenoble, FRA)

Future X-ray science at modern synchrotrons and X-ray Free Electron Lasers will bridge the gap between visible light and electron microscopy. The European Synchrotron Radiation Facility (ESRF) has launched an ambitious and innovative upgrade through the Extremely Brilliant Source (EBS) project to become the first high-energy fourthgeneration synchrotron facility. Other facilities around the world will follow. If exploited with high-performance and tailored instrumentation, the highly brilliant and coherent beams produced by such photon sources will make possible new research opportunities in nano-science and nano-technologies, complex biological and soft-matter systems, time-resolved dynamical processes in complex materials, matter at extreme conditions, and in X-ray imaging techniques and applications. In this context, critically better X-ray detection is required, and a worldwide effort is put in pursuing or launching detector development programmes. Among these, the ESRF-EBS Detector Development Plan consists of a coherent combination of projects and activities including the prototyping of completely new advanced detector systems, in collaboration with other laboratories, as well as further developing detection related technologies that will be instrumental to maintaining the high overall level of quality and scientific productivity of the beamlines. The latest results obtained in the framework of this detector development plan will be presented, along with the outcomes of complementary developments at other facilities, laboratories or companies. The talk will be focused on pixelated X-ray detection solutions for high energy and high photon fluxes applications, using high-Z sensor technologies and new readout ASIC design. 